\title{
Critical-doping universality for cuprate superconductors: Oxygen nuclear-magnetic-resonance investigation of $\left(\mathrm{Ca}_{x} \mathbf{L a}_{1-x}\right)\left(\mathrm{Ba}_{1.75-x} \mathbf{L a}_{0.25+x}\right) \mathrm{Cu}_{3} \mathrm{O}_{y}$
}

\author{
Eran Amit and Amit Keren \\ Physics Department, Technion, Israel Institute of Technology, Haifa 32000, Israel
}

\begin{abstract}
The critical doping levels in cuprates, where the ground state changes its nature (from an antiferromagnet to a spin glass to superconductor to metal), are not universal. We investigate the origin of these critical doping variations by measuring the in-plane oxygen $p_{\sigma}$ hole density in the $\mathrm{CuO}_{2}$ layers as a function of the oxygen density $y$ in $\left(\mathrm{Ca}_{x} \mathrm{La}_{1-x}\right)\left(\mathrm{Ba}_{1.75-x} \mathrm{La}_{0.25+x}\right) \mathrm{Cu}_{3} \mathrm{O}_{y}$. This is done using the oxygen 17 nuclear quadrupole resonance parameter $\nu_{Q}$. We compare compounds with $x=0.1$ and 0.4 which have significant critical $y$ variations and find that these variations can be explained by a change in the efficiency of hole injection into the $p_{\sigma}$ orbital. This allows us to generate a unified phase diagram for the CLBLCO system across the entire doping range, with no adjustable parameters.
\end{abstract}

There are several critical doping levels in the phase diagram of the cuprates where the ground state changes [1]. The first critical doping level is when the long range antiferromagnetic (AFM) order is destroyed and replaced by a spin glass (SG) state; next superconductivity (SC) emerges; then the spin glass is destroyed; and finally, superconductivity is destroyed and replaced by a metallic state. These critical levels exist in the phase diagram of all cuprates which can be doped over a wide range such as $\mathrm{La}_{2-x} \mathrm{Sr}_{x} \mathrm{CuO}_{4}$ (LSCO) and $\mathrm{YBa}_{2} \mathrm{Cu}_{3} \mathrm{O}_{y}(\mathrm{YBCO})$, but they vary between compounds. Several attempts have been made to construct a universal phase diagram but thus far only partial diagrams, of only one or two phases, have been achieved [2 6]. One particular example is the phase diagram of the $\left(\mathrm{Ca}_{x} \mathrm{La}_{1-x}\right)\left(\mathrm{Ba}_{1.75-x} \mathrm{La}_{0.25+x}\right) \mathrm{Cu}_{3} \mathrm{O}_{y}$ (CLBLCO) system shown in Fig. 1(a), which includes four different families with $x=0.1,0.2,0.3$ and 0.4 [7]. This phase diagram clearly demonstrates that the critical oxygen densities $y$ depend on $x$ and thus are not universal.

The reason for lack of universality is not clear and could be one of many. For example, it is possible that the doping efficiency of the $\mathrm{CuO}_{2}$ planes is family-dependent. A second option is interlayer coupling, which in CLBLCO is $x$ dependent [7]; it is conceivable that the interlayer coupling determines the critical doping. Another possibility is that two different kinds of holes are formed in the $\mathrm{CuO}_{2}$ planes, and only the "mobile holes" participate in the SC mechanism; perhaps the level of mobility varies between families [9, 10]. Finally, in the $t-J$ model the critical doping where the AFM order is destroyed depends on $t / J$ [11]; it could be that $t / J$ varies between CLBLCO families. In this work we investigate the origin of the critical doping level variation between different CLBLCO families by directly measuring the hole density in the $\mathrm{CuO}_{2}$ plane of CLBLCO using the oxygen Nuclear Quadrupole Resonance (NQR) parameter ${ }^{17} \nu_{Q}$. This parameter is extracted from Nuclear Magnetic Resonance (NMR) experiments and is directly related to the density of holes in the in-plane oxygen $p_{\sigma}$ orbital as we demon-

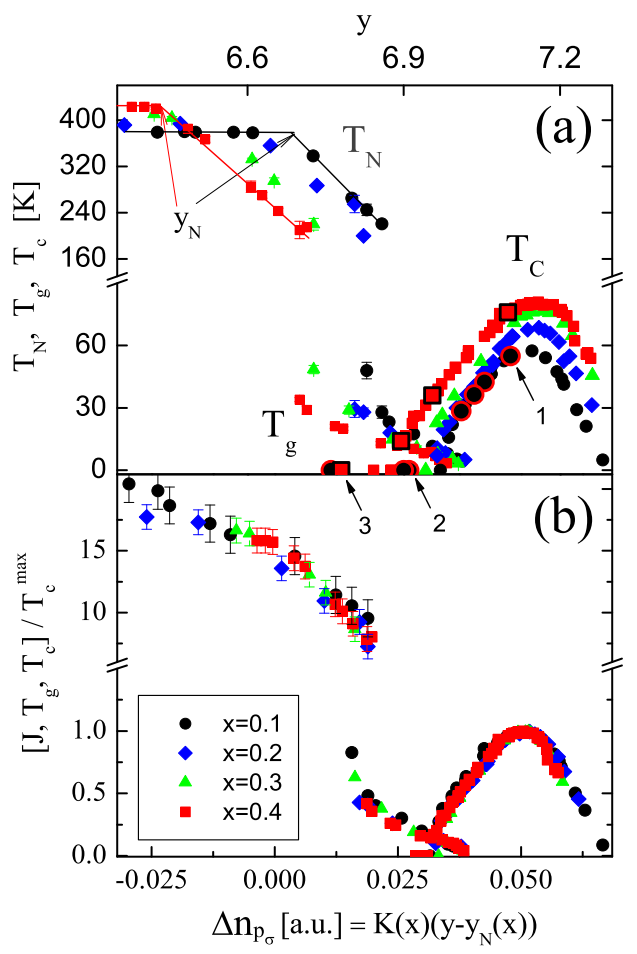

FIG. 1. (color online) $\left(\mathrm{Ca}_{x} \mathrm{La}_{1-x}\right)\left(\mathrm{Ba}_{1.75-x} \mathrm{La}_{0.25+x}\right) \mathrm{Cu}_{3} \mathrm{O}_{y}$ phase diagram (a) showing the superconductivity, Neél, and glass critical temperatures $T_{c}, T_{N}$ and $T_{g}$ respectivly as a function of $x$ and $y$ [7]. The arrows mark the oxygen densities where $T_{N}$ starts to decrease. Fig. (b) is obtained from (a) as described in the text.

strate below.

Our investigation concentrates on CLBLCO since its phase diagram is smooth and systematic. It also does not have abrupt features such as the kink in the LSCO SC dome at $x=0.125$ [12], or a structural phase transition and chain ordering as in YBCO. In fact, CLBLCO has a tetragonal structure (similar to underdoped YBCO) for all values of $x$ and $y$. Consequently, the layer equivalent to the "chain layer" of the YBCO can hold oxygen in both the $a$ and $b$ directions. Therefore, for each family, 
the parameter $y$ varies between 6.4 and 7.25 and controls the doping level. The crystal quality and the total cation charge are also $x$-independent [14]. These properties reduces the number of variable determining $T_{c}$. Thus, understanding superconductivity in this compound could shed light on all other cuprates.

As $x$ increases from $x=0.1$ to $x=0.4$ the maximum $T_{c}\left(T_{c}^{\max }\right)$ increases from 57 to $81 \mathrm{~K}$; the glass temperature $T_{g}$ decreases; and the Neél temperature $\left(T_{N}\right)$ of the parent compound increases from 380 to $425 \mathrm{~K}$. Another important feature of CLBLCO is that for each family there is an oxygen density which marks a transition between a constant $T_{N}$ and a decreasing $T_{N}$ as a function of $y$, as demonstrated in Fig. 1(a). We denote this density as $y_{N}$; for the $x=0.1,0.2,0.3,0.4$ families $y_{N}=6.69,6.63,6.52,6.43$, respectively.

Due to the systematic behavior of CLBLCO, the four different phase diagrams in Fig. 1(a) can be reduced into one unified diagram in three steps [7, 8]: 1. Extracting the values of the in-plane AFM coupling $J$ from $T_{N}$ for each family by dividing out the interplane coupling contribution. 2. Dividing $J, T_{g}$, and $T_{c}$ of each family by $T_{c}^{\max }$ of that family. 3 . Stretching the oxygen density axis for each family around its $y_{N}$ by a factor $K(x)$, namely, introducing the quantity $K(x)\left(y-y_{N}\right)$, where $K=0.113,0.098,0.079,0.069$, for the $x=0.1$ to 0.4 families respectively. The resulted scaled phase diagram is shown in Fig. 1(b). The scaling procedure is somewhat different from that in previous works where the stretching was done around the oxygen density of $T_{c}^{\max }$ and a different set of $K \mathrm{~s}$ was used [7, 14 16]. Multiplying all the $K \mathrm{~s}$ by a numerical factor would yield equally good data collapse. Here the $K$ s are chosen so that optimal doping is at $K(x)\left(y-y_{N}\right)=0.05$, for reasons that will become clear below. The ratio of $K$ between the $x=0.4$ and $x=0.1$ families is $1: 1.62$. Using the scaling terminology the question we address experimentally in this work is: can $K(x)$ be explained by the in-plane oxygen $p_{\sigma}$ orbital doping efficiency, or is some more exotic explanation required?

For our experiments, sintered pellets of CLBLCO with different $x$ values were prepared using standard techniques [17], and then ground into powder. Since only ${ }^{17} \mathrm{O}$ has nuclear spin but its natural abundance is only $0.038 \%$, the samples were enriched with this isotope. In the enrichment process the samples were heated to $520^{\circ} \mathrm{C}$ for five days and then cooled to $320^{\circ} \mathrm{C}$ for five more days in enriched oxygen gas with an isotope fraction of $40-50 \%$. In order to obtain different oxygen levels some of the enriched samples were later annealed in either natural oxygen or nitrogen environments at different temperatures for 24 hours, and then quenched in liquid nitrogen. When possible, $T_{c}$ was determined with a magnetometer as shown in the inset of Fig. 2. We used cryogenic SQUID, at a field of 5Oe in FC conditions. In this inset the normalized magnetization of four samples with $x=0.1$ and $y=7.105,7.055,7.035,7.01$ is shown, demonstrating the variation in $T_{c}$. The oxygen density was obtained from the known CLBLCO phase diagram (Fig. 11). For the very underdoped samples, which are not superconducting (see samples 1 and 2 in Fig. 1), the oxygen density was determined by iodometric titration [18].

In our NMR experiment all the eleven samples emphasized in Fig. 1(a) by enlarged symbols were measured. We used constant frequency $f=36.525 \mathrm{MHz}$ and temperature of $110 \mathrm{~K}$ (the dependence of $\nu_{Q}$ on temperature is within our experimental error [19]). Measurements were performed over a range of external magnetic fields. For each field 20480 spin echo sequences were collected. The intensity for each field is the integral over the Fourier transform of the raw data.

There are three oxygen sites in CLBLCO: the planar $\mathrm{O}_{23}$, apical $\mathrm{O}_{4}$ and "chain" $\mathrm{O}_{1}$. The NMR line of the $\mathrm{O}_{1}$ site is negligible [20]. The apical oxygen does not affect the measured NMR lineshape either. We measure at $110 \mathrm{~K}$, where its intensity is much smaller than the planar oxygen and its line is wider 20]. As a result, the spectrum is dominated by only one oxygen nucleus instead of three. However, the ${ }^{139} \mathrm{La}$ nucleus appears in some of the measurements when the amount of ${ }^{17} \mathrm{O}$ in the sample is small. In our working frequency the ${ }^{139} \mathrm{La}$ has a central transition at $6.1 \mathrm{~T}$, while the ${ }^{17} \mathrm{O}$ central transition is at $6.33 \mathrm{~T}$. Therefore, in samples with a low enough concentration of ${ }^{17} \mathrm{O}$ the lanthanum signal changes the NMR line shape at low fields, but not at high fields.

We present in Fig. 2 NMR lines of three samples: (1) $x=0.1$ close to optimal doping, (2) $x=0.1$ underdoped, and (3) $x=0.4$ very under doped. Their place on the phase diagram is indicated in Fig. 1(a). There is a clear difference around $6.43 \mathrm{~T}$ between lines (1) and (3) but lines (2) and (3) are similar. As we explain below, this is a consequence of different $p_{\sigma}$ densities in samples (1) and (3), and similar densities in samples (2) and (3). In the lower field regime of the underdoped sample (2) the lanthanum signal dominates the spectrum.

A quadrupole nucleus such as ${ }^{17} \mathrm{O}$ can be viewed as a non spherical charge distribution whose energy depends on its orientation with respect to the internal electric fields. The nuclear Hamiltonian in an external magnetic field $\mathbf{H}$ is a sum of the usual Zeeman interaction and additional quadrupole term, and is given by

$$
\mathcal{H}=-\gamma \hbar \mathbf{H}(\mathbf{1}+\sigma) \mathbf{I}+\frac{e Q V_{z z}}{4 I(2 I-1)}\left[3 I_{z}^{2}-I^{2}+\eta\left(I_{x}^{2}-I_{y}^{2}\right)\right]
$$

where ${ }^{17} \gamma=5.77 \mathrm{MHz} / \mathrm{T}$ is the gyromagnetic ratio, $\mathbf{I}$ is the nuclear spin operator in the $I=5 / 2$ representation, $\sigma$ is the shift tensor and $e Q$ is the ${ }^{17} \mathrm{O}$ quadrupole moment. The second term is written in the Electric Field Gradient (EFG) system representation where the EFG 


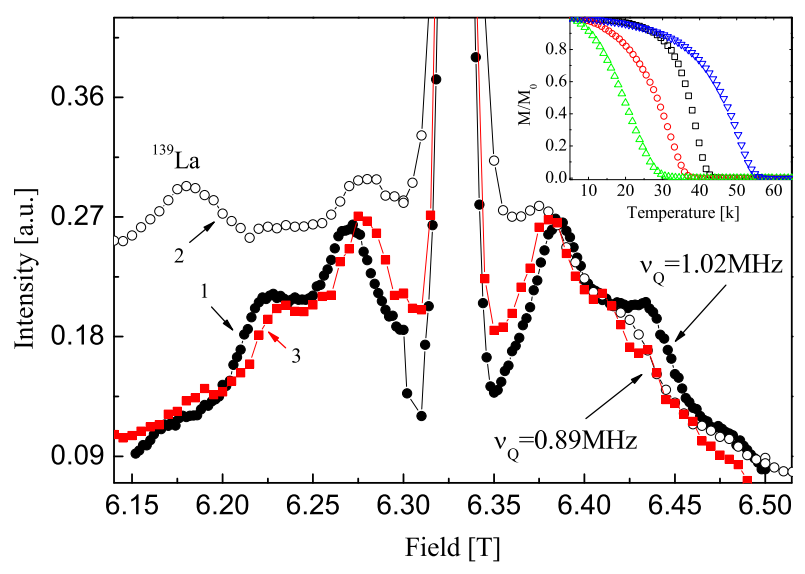

FIG. 2. (color online) Raw NMR data of three samples marked in Fig 1(a): 1 (closed circles) is $\mathrm{x}=0.1$ close to optimal doping $(\mathrm{y}=7.105), 2$ (open circles) is $\mathrm{x}=0.1$ underdoped $(y=6.9), 3$ (closed squares) is $x=0.4$ underdoped $(y=6.79)$. From the high fields data it is clear that the quadrupole frequency of samples 2 and 3 are almost identical but different from sample 1 . The dominant contribution in sample 2 at lower fields is from the ${ }^{139} \mathrm{La}$ nuclei. The inset shows the temperature dependence of the normalized magnetic moment for the $\mathrm{x}=0.1 \mathrm{SC}$ samples with $\mathrm{y}=7.105$ (up triangles), 7.055 (circles), 7.035 (squares) and 7.01 (down triangles).

tensor is diagonal, $V_{z z}=\frac{\partial^{2} V}{\partial z^{2}}$ is the largest (axial) EFG eigenvalue, and $\eta=\left(V_{x x}-V_{y y}\right) / V_{z z}$ is the orthorhombic EFG asymmetry.

In the limit of small quadrupole frequency compared to $\gamma \hbar H$, the energy difference between two nuclear spin states $\Delta E_{m \rightarrow m-1}$ is given by [21]:

$$
\begin{aligned}
\Delta E_{m \rightarrow m-1} & =\gamma h H\left[1-\sigma_{i}\right]-h \nu_{Q}\left[\frac{1}{2}\left(3 \cos ^{2}(\theta)-1\right)\right. \\
& \left.-\frac{1}{2} \eta \sin ^{2}(\theta) \cos (2 \phi)\right]\left(m-\frac{1}{2}\right)
\end{aligned}
$$

where $m$ is the nuclear spin component parallel to the external (Zeeman) magnetic field, $\sigma_{i}=\sigma_{x}+\sigma_{y}+\sigma_{z}$ is the diagonal term of the chemical and Knight shifts (we neglected the off-diagonal terms), and $\theta$ and $\phi$ are the angles between $\mathbf{z}$ and the external magnetic field, and the quadrupole frequency is defined as

$$
\nu_{Q}=\frac{e Q V_{z z}}{4 I(2 I-1)}
$$

A resonance occurs when the frequency $f_{m}\left(H, \nu_{Q}, \sigma_{i}, \eta, \phi, \theta\right)=\Delta E_{m \rightarrow m-1} / h$ equals an applied frequency $f$. In powder, all possible orientations and line broadening must be taken into account.

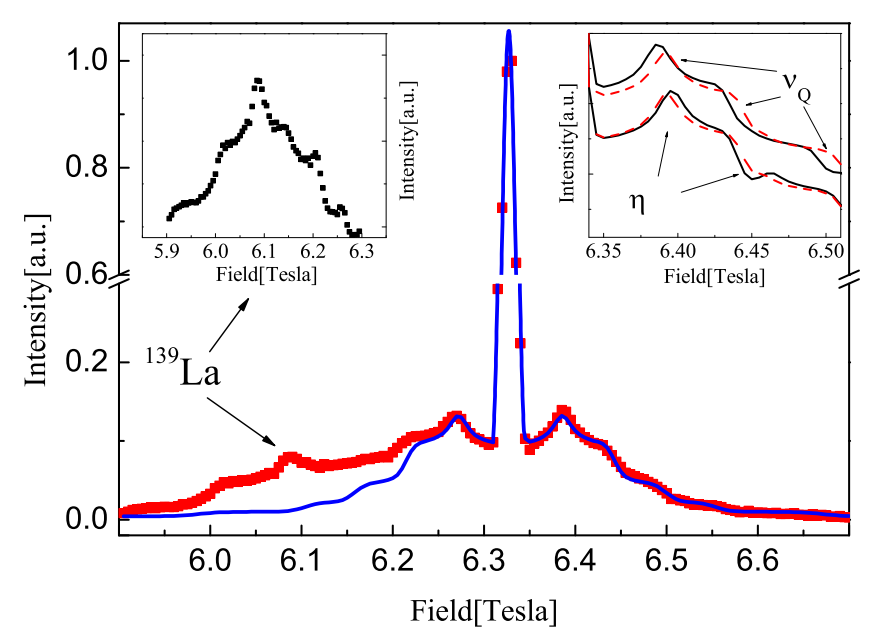

FIG. 3. (color online) Raw data of an NMR measurement (red squares) and a fit of the high field data to Eq. 4 (blue line). At low fields there is a deviation from the raw data due to the ${ }^{139} \mathrm{La}$ line. The difference between the raw data and the fit is presented in the left inset. The right inset contains theoretical plots of samples with two different $\nu_{Q}$ (top lines) and two different $\eta$ (bottom lines), as explained in the text.

Therefore, the spectrum is given by

$$
\begin{aligned}
I(H) & =\sum_{m=-3 / 2}^{5 / 2} W(m) \int_{0}^{\infty} d \sigma_{i}^{\prime} e^{-\frac{\left(\sigma_{i}^{\prime}-\sigma_{i}\right)^{2}}{2 \Delta \sigma_{i}^{2}}} \int_{0}^{\infty} d \nu_{Q}^{\prime} e^{-\frac{\left(\nu_{Q}^{\prime}-\nu_{Q}\right)^{2}}{2 \Delta \nu_{Q}^{2}}} \\
& \int d \Omega \delta\left(f_{m}\left(H, \nu_{Q}^{\prime}, \sigma_{i}^{\prime}, \eta, \phi, \theta\right)-f\right) .
\end{aligned}
$$

The line broadening in our experiments has typical values of $\Delta \nu_{Q}<0.2 \nu_{Q}$ and $\Delta \sigma_{i} \cong 0.002 \sigma_{i}$. Finally $W(m)$ represents the weights of the different transitions and is taken as fit parameter. The effect of the parameter on the line shape is demonstrated in the right panel of Fig. 3, The high field side of the theoretical lines for $\nu_{Q}=0.95$, $1.03 \mathrm{MHz}$ (with $\eta=0.33$ ) and of $\eta=0.28,0.32$ (with $\nu_{Q}=1.02 \mathrm{MHz}$ ) are plotted in the right inset. Arrows mark the regions in which $\eta$ and $\nu_{Q}$ changes have the most effect on the spectrum, and enable us to distinguish between these parameters.

An NMR line of the CLBLCO sample with $x=0.4$ and $y=7.1$ (solid symbols) is presented in the main panel of Fig. 3. The solid line is the best fit of Eq. [4 to the data at the high field side. It gives $\nu_{Q}=0.98 \mathrm{MHz}$ and $\eta=0.33$. These numbers are similar to previous measurements of YBCO [20]. The difference between the fit and the data on the low field side, which is caused by the lanthanum nuclei, is plotted in the left inset. In order to obtain $\nu_{Q}(x, y)$ from all samples we fit Eq. 4 to the NMR spectrum of all the measured samples. The results are shown in Fig. 4 . 
The EFG on a planar oxygen site is induced by the electrons and nuclei surrounding the oxygen [22]. The principal axis of this EFG ( $z$ direction) is parallel to the copper-oxygen-copper axis [20]. The two main contributions to $V_{z z}$ are : I) holes in the oxygen $p_{\sigma}$ orbital; II) holes and nuclei of the atoms surrounding the oxygen. The first contribution is directly proportional to the number of holes created by the doping process. In contrast, (II) has a negligible dependence on doping [23]. Moreover, the holes in the oxygen $p_{\sigma}$ are much closer to the nucleus and therefore their contribution to the EFG is more significant.

The classical formula of $V_{z z}$ is given by $V_{z z}=$ $\int \rho(\vec{r}) \frac{r^{2}-3 z^{2}}{r^{5}} d^{3} r$ where $\rho$ and $r$ are the charge density and distance from the nucleus, respectively. The value of $V_{z z}^{\prime}$ induced by a different charge distribution given by $\rho^{\prime}(\vec{r})=\xi^{3} \rho(\xi \vec{r})$, where $\xi$ is a constant, is

$$
V_{z z}^{\prime}=\xi^{3} V_{z z}
$$

The oxygen $p_{\sigma}$ electronic wave functions of the different CLBLCO samples differ mostly in their typical length-scale. The characteristic length is proportional to the unit cell parameter $a$. Neutron diffraction experiments show that $a$ changes only by about one percent between the different families, and by 0.1 percent within a family [15]. Therefore, using Eq. 5 and Eq. 3 it is expected that $\Delta\left(V_{z z} a^{3}\right) \propto \Delta\left(\nu_{Q} a^{3}\right) \propto \Delta n_{p_{\sigma}}$, where $n_{p_{\sigma}}$ is the hole density in the oxygen $p_{\sigma}$ orbital and $\Delta$ stands for the changes induced by the doping process.

In Fig. 4 we present $\nu_{Q} a^{3}$ versus oxygen levels $y$ for the two families with $x=0.1$ and 0.4 (Samples 1, 2 and 3 are the same as in Fig. 1 and 21). We can clearly see that the rate at which $\nu_{Q} a^{3}$ increases with increasing $y$ varies between the two families. The data from the different families generate two different linear curves. Two straight lines are fitted to these datasets with the constraint that the slopes ratio is 1.62 , which, as mentioned before, is the ratio of $K(0.4)$ to $K(0.1)$. The measured $\nu_{Q} a^{3}$ versus $y$ can be explained well by the two lines. When we perform a linear fits with no constrains the slopes ratio is $2.2 \pm 0.65$ which is within error bars equal to the $K$ 's ratio. This is the main experimental finding of this work.

Since we have demonstrated experimentally that $\Delta\left(\nu_{Q} a^{3}\right) \propto K(x)\left(y-y_{N}\right)$ and argued above that $\Delta\left(\nu_{Q} a^{3}\right) \propto \Delta n_{p_{\sigma}}$, we conclude that

$$
\Delta n_{p_{\sigma}} \propto K(x)\left(y-y_{N}\right) .
$$

Therefore, the doping efficiency of the $p_{\sigma}$ orbital is family dependent. We would like to emphasize again that we can only quantify the doping efficiency ratio between the two different families ( $K$ s ratio), but not their absolute value. Hence the proportionality sign in Eq. 6. The set of $K \mathrm{~s}$ which generate Fig. प1(b) are chosen to give $\Delta n_{p_{\tau}}=$ 0.05 at optimal doping according to Hasse et al. [23].

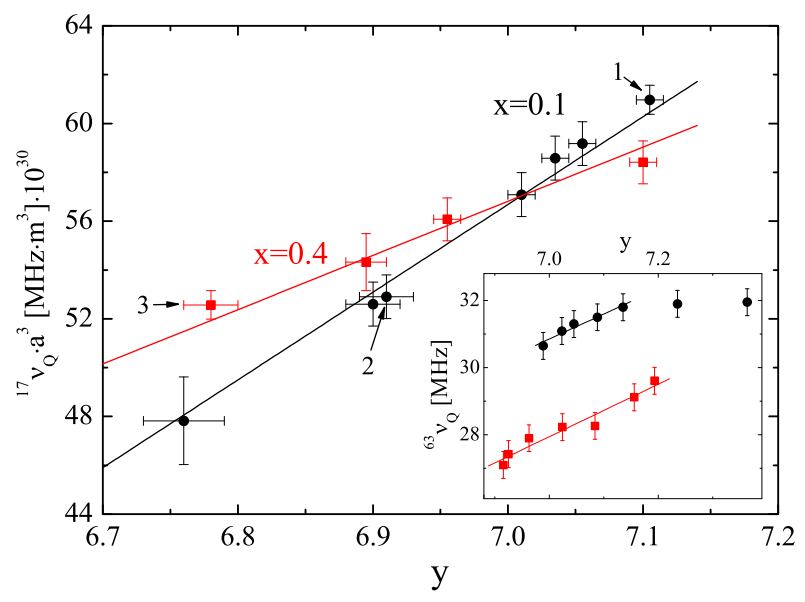

FIG. 4. (color online) The translation of the number of oxygen atoms per unit cell (abscissa) into the number of $p_{\sigma}$ holes (ordinate), as extracted from the NMR data. The $\mathrm{x}=0.1$ family is in black circles, and the $\mathrm{x}=0.4$ family is in red squares. The ratio between the slopes is equal to the stretching ratio between the families in the scaling process shown in Fig. 11 (see text). The place on the phase diagram of samples 1 to 3 is shown in Fig. 1(a), and their raw NMR data is depicted in Fig. 2 The inset shows that measurements of the copper quadrupole frequencies in CLBLCO give the same slope for both families [9].

Negative values of $\Delta n_{p_{\sigma}}$ represent $\mathrm{CuO}_{2}$ planes which are not doped. Another degree of freedom is the number of $p_{\sigma}$ holes for $y<y_{N}$. Following Ref. [23] again, by setting $n_{p_{\sigma}}\left(y<y_{N}\right)=0.11$ we obtain $n_{p_{\sigma}}=0.16$ at optimal doping.

There were several attempts in the past to find a relation between the number of holes in the $\mathrm{CuO}_{2}$ plane and oxygen level $y$ of CLBLCO. Chmaissem et al. used bond valence summation (BVS) calculations based on structural parameters determined by neutron diffraction 24]. Keren et al. measured the in plane ${ }^{63} \mathrm{Cu} \mathrm{NQR}$ parameter ${ }^{63} \nu_{Q}$ which is shown in the inset of Fig. 4. and Sanna et al. experimented with x-ray fine structure (XFS) [10]. BVS has some theoretical arbitrariness and is not completely reliable. ${ }^{63} \nu_{Q}(y)$ shows no family dependence because ${ }^{63} \nu_{Q}$ is sensitive to charge on the apical $\mathrm{O}_{4} p_{\sigma}, \mathrm{Cu} 3 d_{x^{2}-y^{2}}, 3 d_{z^{2}-r^{2}}$ and $4 s$, and $\mathrm{O}_{2,3} p_{\sigma}$ holes simultaneously [23]; hence it is not an ideal probe and a difference in the slopes of ${ }^{63} \nu_{Q}(x, y)$ could not be detected within the experimental error bars. Finally, the XFS peak is constructed of three contributions [10] which again limit their resolution. Therefore, none of the three attempts could find a difference in the doping efficiency of the planes within experimental resolution. The oxygen NQR has the advantage of measuring directly the dependence of $p_{\sigma}$ hole density $n_{p \sigma}$ on the oxygen level $y$, and, indeed, this probe detects variations in doping efficiency. 
The physical meaning of Fig. 4 and Eq. 6 is that the efficiency of the doping process, namely the injection of holes into the oxygen $p_{\sigma}$ orbital, varies between the CLBLCO families. Moreover, the scaling procedure leading from Fig. 1(a) to Fig. 1(b) can now be fully justified: the third step is needed because the oxygen density is not the relevant parameter and one must use $n_{p_{\sigma}}$ in the phase diagram. The second step means that $T_{c}^{\max }$ is determined by the magnetic super-exchange interaction energy scale $J$, namely, $J(x) / T_{c}^{\max }(x)$ is $x$ independent. This leads to the unified phase diagram of Fig. 1(b) which is obtained with no adjustable parameters.

This work was funded by the Israeli Science Foundation.

[1] P. A. Lee, Rep. Prog. Phys. 71, 012501 (2008).

[2] Y. J. Uemura et al., Phys. Rev. Lett. 62, 2317 (1989).

[3] C. C. Homes et al., Nature 430, 539 (2004).

[4] T. Honma, and P. H. Hor, Phys. Rev. B 77, 184520 (2008).

[5] A. Mourachkine, Jour. of Superconductivity 17, 269 (2004).

[6] H. Zhou, Z. Ying, M. Cuoco, and C. Noce,
arXiv: 1002.4281 v 1

[7] R. Ofer et al., Phys. Rev. B 74, 220508(R) (2006).

[8] A. Kanigel et al., Phys. Rev. Lett. 88, 137003 (2002).

[9] A. Keren, A. Kanigel, and G. Bazalitsky, Phys. Rev. B. 74, 172506 (2006).

[10] S. Sanna et al., EPL 86, 67007 (2009).

[11] M. Calandra, F. Becca and S. Sorella, Phys. Rev. Lett. 81, 5185 (1998).

[12] H. Takagi et al., Phys. Rev. B. 41, 1863 (1990).

[13] J. D. Jorgensen et al., Phys. Rev. B. 40, 4454 (1989).

[14] Amit Keren, New J. Phys. 11065006 (2009).

[15] R. Ofer, A. Keren, O. Chmaissem and A. Amato, Phys. Rev. B 78, 140508(R) (2008).

[16] Y. Lubashevsky and A. Keren, Phys. Rev. B 78, 020505(R) (2008).

[17] D. Goldschmidt et al., Phys. Rev. B 48, 532 (1993).

[18] E.H. Appelman et al., Inorg. Chem 26, 3237 (1987).

[19] B. Grevin, Y. Berthier and G. Collin, Phys. Rev. Lett. 85, 1310 (2000).

[20] E. Oldfield et al., Phys. Rev. B 40, 6832 (1989).

[21] J. F. Baugher, P. C. Taylor, T. Oja, and P. J. Bray, Jour. of Chem. Phys. 50, 4914(1969).

[22] C. P. Slichter, Principles of Magnetic Resonance (Harper and Row, New York, 1963).

[23] J. Haase, O. P. Sushkov, P. Horsch, and G. V. M. Williams, Phys. Rev. B 69, 094504 (2004).

[24] O. Chmaissem, Y. Eckstein, and C. G. Kuper, Phys. Rev. B 63, 174510 (2001). 